\title{
ВMJ Global Health Design of a randomised intervention study: the effect of dumbbell exercise therapy on physical activity and quality of life among breast cancer survivors in Malaysia
}

\author{
Adamu Ahmad Rufa'i, ${ }^{1,2}$ Wan Abdul Manan Wan Muda, ${ }^{1}$ Siew Hwa Yen, ${ }^{3}$ \\ Aishah Knight Abd Shatar, ${ }^{4}$ Bhavaraju Venkata Krishna Murali, ${ }^{5}$ Shu Wen $\operatorname{Tan}^{1}$
}

To cite: Rufa'i AA, Muda WAMW, Yen SH, et al. Design of a randomised intervention study: the effect of dumbbell exercise therapy on physical activity and quality of life among breast cancer survivors in Malaysia. BMJ Global Health 2016;1: e000015. doi:10.1136/ bmjgh-2015-000015

Received 23 November 2015 Revised 24 June 2016 Accepted 1 July 2016

\section{CrossMark}

\footnotetext{
${ }^{1}$ School of Health Sciences, Universiti Sains Malaysia, Kota Bharu, Kelantan,

Malaysia

${ }^{2}$ Department of Physiotherapy, College of Medical Sciences, University of Maiduguri, Borno, Nigeria ${ }^{3}$ School of Distance Education, Universiti Sains Malaysia, Pinang, Malaysia

${ }^{4}$ Advanced Medical and Dental Institute, Universiti Sains Malaysia, Kepala Batas, Penang, Malaysia ${ }^{5}$ Department of Oncology, Hospital Universiti Sains Malaysia, Kota Bharu, Kelantan, Malaysia
}

Correspondence to Adamu Ahmad Rufa'i; adamuarufai@gmail.com

\section{ABSTRACT}

Background: Participation in physical activity has a positive impact on the overall health and quality of life, whereas physical inactivity is associated with a poor prognosis among breast cancer survivors. Despite the health-enhancing benefits of physical activity, the majority of Malaysian breast cancer survivors are not physically active. This paper presents the design of a randomised study to evaluate the feasibility and effect of exercise therapy intervention using light resistance dumbbell exercise to promote active lifestyle and improve the quality of life of breast cancer survivors in Malaysia.

Methods/design: This is an intervention study of a 12-week exercise therapy that will explore and compare the effects of light resistance and aerobic exercise on physical activity level and quality of life components in 102 female breast cancer survivors. Major eligibility criteria include histologically confirmed diagnosis of breast cancer stages I-III, 3-12 months postdiagnosis, and absence of any disorder contraindicating exercise. Participants will be stratified based on menopausal status (pre-menopause vs postmenopause) and then assigned randomly to one of three groups. Participants in group A will participate in a three-times weekly supervised resistance exercise using light resistance dumbbells; participants in group B will participate in a three-times weekly supervised aerobic exercise; while participants in group $\mathrm{C}$ (control group) will be given aerobic exercise after completion of the intervention. The primary end points include physical activity level and quality of life components. The secondary end points are body mass index, body composition, total caloric intake, and waist-to-hip ratio. Discussion: Although there have been many studies of resistance exercise in breast cancer survivors, this is the first study using this specific mode of resistance. Findings will contribute data on the feasibility and effects of light resistance dumbbell exercises, and provide knowledge on the physical activity intervention programme that will maximally promote better overall health and well-being of survivors.

\section{INTRODUCTION}

Breast cancer remains the most common cancer affecting women world-wide, with approximately 1.7 million persons identified with the disease in $2012 .^{1-3}$ This may be due to the rising incidence and improved survival rates. Globally, the incidence rate has been estimated at 43 per 100000 women, with 5- and 10-year survival rates of $90 \%$ and $84 \%$, respectively. ${ }^{1}$ The number of breast cancer survivors is rising, as is evident from recent data showing an annual decrease in mortality by $2.2 \%$ and a $0.1 \%$ increase in incidence rates from 1990 to 2008. The increasing rate of breast cancer survivors is primarily attributable to the technological advancements in medical practice which enhances early detection as well as better treatment options. On the other hand, ageing and poor lifestyle such as physical inactivity and unhealthy dietary patterns have been implicated as the main causes for the rising incidence. ${ }^{2} 3$

Breast cancer survivors continue to attract public health attention because of their special healthcare needs. This growing population faces several challenges from the condition and the unwanted secondary effects of its management. Thus, it is obvious that breast cancer survivors will present with various needs along the survivorship continuum. Unfortunately, the survivorship phase of cancer care has not been accorded the attention it deserves; the healthcare system gives more attention to the 'cure' compared to the post-diagnosis and treatment effects. Some of the common effects include fatigue, functional deterioration, sleeplessness, and decline in quality of life. ${ }^{45}$ Other effects include the risk of cancer recurrence, associated chronic diseases, and 
psychological disturbance. ${ }^{6}$ Challenges unique to breast cancer survivors include limited range of arm motion, ${ }^{7}$ lymphoedema, ${ }^{8}$ impaired cognitive function, ${ }^{9}$ and osteoporosis. ${ }^{10}$ In addition, earlier studies in the literature have shown that breast cancer treatments, chemotherapy in particular, result in fatigue, bodyweight gain, excess adipose tissue, and lack of physical activity. ${ }^{11}{ }^{12}$ Similarly, adjuvant chemotherapy predisposes breast cancer patients, especially pre-menopausal women, to increased central obesity and body mass index (BMI) which is harmful to their life. ${ }^{13}$ Another effect of breast cancer treatment is the decrease in the total caloric intake. ${ }^{14}$

These effects are interrelated and can contribute to a pronounced deterioration in the condition of the patient. For example, increased chances of breast cancer recurrence have been reported among overweight patients and/or those who increased their weight postdiagnosis. ${ }^{15}$ Also, fatigue, which affects about $70 \%$ of cancer patients during chemotherapy/radiotherapy, is considered to occur as a result of the effects of the disease and its treatments. ${ }^{16}$ Fatigue is identified as the main factor responsible for a decline in physical activity participation among cancer patients, with a consequent change in overall health and quality of life. ${ }^{17}$ In addition, studies have demonstrated that the inactive way of life adopted by cancer survivors is significantly related to unhealthy changes in body composition. ${ }^{18}$ All of these factors are detrimental and could lead to the emergence of chronic diseases because metabolic and immunological responses have already been compromised in these patients. ${ }^{19}$ Hence it is critical to help breast cancer patients faced with these myriad of problems that are unfavourable to their survival.

One of the established non-pharmacologic means of preventing and/or alleviating these effects is physical activity, which is also a very useful element of a healthy lifestyle. ${ }^{6}$ Among its many advantages, physical activity has been shown to have a positive impact on the physiological outcomes involving a strong and healthy cardiovascular system, improved physical functioning, an effective immune system, muscle vitality, balanced body configuration, and diminished nausea and fatigue. In addition, physical activity enhances psychological wellbeing such as emotional state, self-esteem, body image, and depression that occurs usually after the disease has been identified. ${ }^{20-22}$ Physical activity also helps improve the diminished total caloric intake commonly associated with cancer treatment. ${ }^{23}$ Whether carried out in a planned fashion such as exercise or as a result of daily activities, physical activity has the potential of boosting general health and improving mental state as well as quality of life. ${ }^{24}$ However, despite these health benefits, the majority of breast cancer survivors are not physically active. ${ }^{25}$ Traditionally, cancer patients are advised by their attending clinicians to have a rest and not engage in physical activity. Nowadays, this approach has been challenged by evidence emerging from several studies on the benefits of exercise. These studies have indicated that exercise is safe both during and after cancer treatment. ${ }^{6}$

The American College of Sports Medicine guidelines emphasise the promotion of physical activity to improve cancer outcomes among cancer survivors. ${ }^{6}$ In the guidelines, physical activity has been shown to benefit breast cancer survivors in addition to its association with decreased risk of having the disease. Physical activity interventions usually comprise training in the form of aerobic or resistance exercise, or a combination of these exercises. Aerobic exercise training enhances the cardiorespiratory performance ${ }^{26} 27$ whereas resistance exercise training enhances muscle strength, bone density and body balance that eventually reduce the risks of having injuries. ${ }^{28}{ }^{29}$ Nevertheless, interest concerning the safety of upper body exercise, which requires much effort such as resistance exercise among breast cancer survivors susceptible to lymphoedema, has led to a number of randomised controlled trials. ${ }^{30-32}$ Interestingly, a recent systematic review and meta-analysis found resistance exercise training reduced the incidence/exacerbation of lymphoedema among breast cancer patients. ${ }^{33}$ Numerous intervention studies have administered aerobic and resistance exercise training in breast cancer patients. However, most of these studies have focused on the effects on survival and other supportive care end points. Few of them gave attention to physical activity participation among the breast cancer patients. Furthermore, these studies mostly utilised expensive and sophisticated equipment for the resistance exercise training. Such expensive and difficult to operate machines may not be available in some health facilities. Therefore, there is a need for a simple and easy to use means of intervention for health promotion, to offer personalised exercise training programmes.

In this preliminary study, we propose a dumbbell exercise developed and promoted by the late Emeritus Professor Masashige Suzuki (1940-2011) of Waseda University, Japan in 1983. ${ }^{34} 35$ The dumbbell exercise (in the form of light resistance) offers moderate to vigorous intensity exercise. Since its introduction, the use of this dumbbell exercise has spread across Japan, and some parts of China, Korea, and Malaysia. Currently, a 10-year prospective study is in progress on the effects of the dumbbell exercise on the prevention of sarcopenia among the elderly population in Korea. ${ }^{36}$ Each dumbbell is made up of fabric material filled with $300 \mathrm{~g}$ of brown rice; thus a pair of dumbbells weighs $600 \mathrm{~g}$. The dumbbell exercise comprises 12 movements that exercise the large muscle groups of the lower and upper body parts. ${ }^{35} 36$ The dumbbell exercise training works on the shoulders, chest, biceps and triceps, the back and abdominal muscles, as well as the thigh and calf. ${ }^{37}$ Compared to metal dumbbells, this dumbbell offers a better grip and is safe and easier to use. ${ }^{36}$ The dumbbell exercise has positive beneficial effects for all people irrespective of age. Some of the benefits include prevention of sarcopenia, osteopenia, weight control and 
management, and overall health promotion. Studies have been carried out on the effects of light resistance exercise using dumbbells on resting metabolic rate, diet-induced thermogenesis and body composition, ${ }^{35}$ fat reduction, ${ }^{36}$ body composition and physical fitness, ${ }^{37}$ concentrations of amino acids, ${ }^{38} 39$ and weight reduction, ${ }^{40}$ and the results were found to be promising. However, to the best of our knowledge, there have been no such studies in Malaysia, particularly among breast cancer survivors. Therefore, the present study will be the first to use light resistance dumbbells to offer individualised exercise training among breast cancer survivors in Malaysia.

This preliminary study is a prospective randomised intervention in breast cancer survivors who are receiving chemotherapy and/or radiotherapy. The primary aim of the study is to investigate the effects of a 12-week light resistance dumbbell exercise therapy intervention, and compare the effects of light resistance dumbbell and static bicycle ergometer exercise training, on physical activity levels and quality of life components involving physical, social, functional, and emotional well-being domains. The secondary aims are to determine the effects of exercise on BMI, body composition, total caloric intake, and waist-to-hip ratio. Because of the preliminary nature of the study, the safety and feasibility of the exercise programme will be assessed, and adherence will be evaluated to determine the sustainability of the effects.

\section{METHODS}

\section{Study design}

This is a prospective single blind randomised intervention study, the details of which are depicted in figure 1 . Briefly, the intervention involves women with histologically confirmed stages I-III breast cancer receiving either chemotherapy, radiotherapy, or both, within 3-12 months after diagnosis. An informed written consent will first be obtained from the participants; thereafter they will be randomised to exercise therapy groups (A or B) or a control group (C). Assessment of the intervention end points will be conducted at baseline $\left(\mathrm{T}_{0}\right)$, after a 12-week intervention $\left(\mathrm{T}_{1}\right)$, and after 12 weeks of follow-up $\left(T_{2}\right)$, as shown in figure 2. Furthermore, this intervention study will offer a 4-week exercise session to the control group after the study period, to enhance the participation rate and ensure high compliance with the intervention. The groups will be monitored throughout the study by a qualified exercise expert in order to ensure smooth execution of this tailored and individualised exercise programme.

\section{Participants}

For the purpose of this study the definition of a 'cancer survivor' was adopted from the National Coalition for Cancer Survivorship which defined a cancer survivor as "any individual that has been diagnosed with cancer, from the time of discovery and for the balance of life". ${ }^{41}$ Female breast cancer survivors who are not regularly active (ie, who exercise $<3$ times/week, $<30 \mathrm{~min}$ in duration), with BMI $\geq 25 \mathrm{~kg} / \mathrm{m}^{2}$ or body fat $>30 \%$, and have fulfilled the inclusion criteria will be eligible, provided they have passed the health and fitness screening. A valid and reliable interviewer-administered physical activity questionnaire will be used to assess the physical activity history at baseline. Table 1 presents the inclusion and exclusion criteria to be used for recruitment of the participants.

\section{Setting}

All recruitment and outcome assessments will be done at the oncology clinic of Hospital Universiti Sains Malaysia (HUSM), and the intervention programme will be conducted at the Department of Physiotherapy, HUSM.

\section{Recruitment and screening}

Prospective participants will be recruited from the oncology clinic after the study is introduced to the patients by the oncologist. The researcher(s)/research assistant(s) will meet the prospective participants individually during clinic visits for recruitment. During the first contact, prospective participants will be duly counselled about the nature and purpose of the research. They will also be assured that participation is voluntary and all information obtained will only be used for research purposes. Participants who agree to participate in the study will be given an informed consent form. Another convenient day will be arranged to meet with the prospective participants to screen them for health and fitness. Only patients who formally accept to participate in the study by signing the informed consent will be screened.
Figure 1 Flow of the study design. End points assessments are at 0 week (baseline $T_{0}$ ), after 12 weeks (post-intervention $\mathrm{T}_{1}$ ), and after 24 weeks (follow-up $T_{2}$ ).

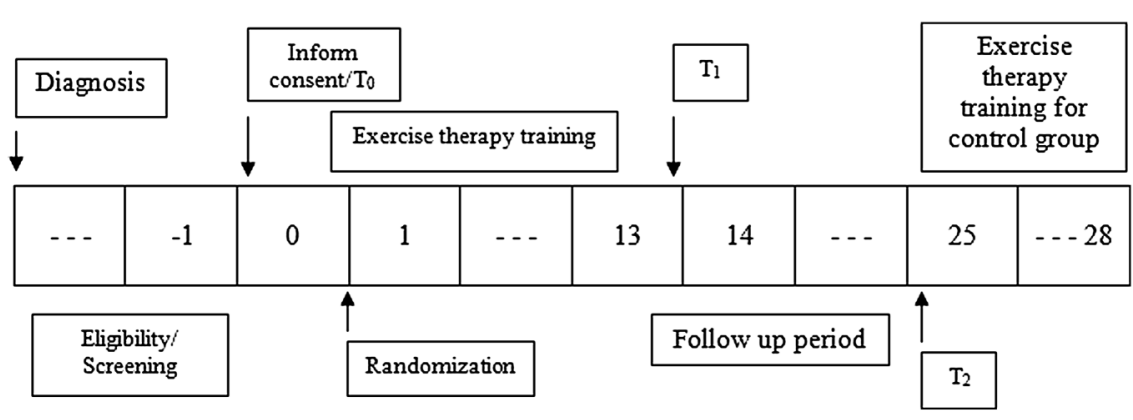




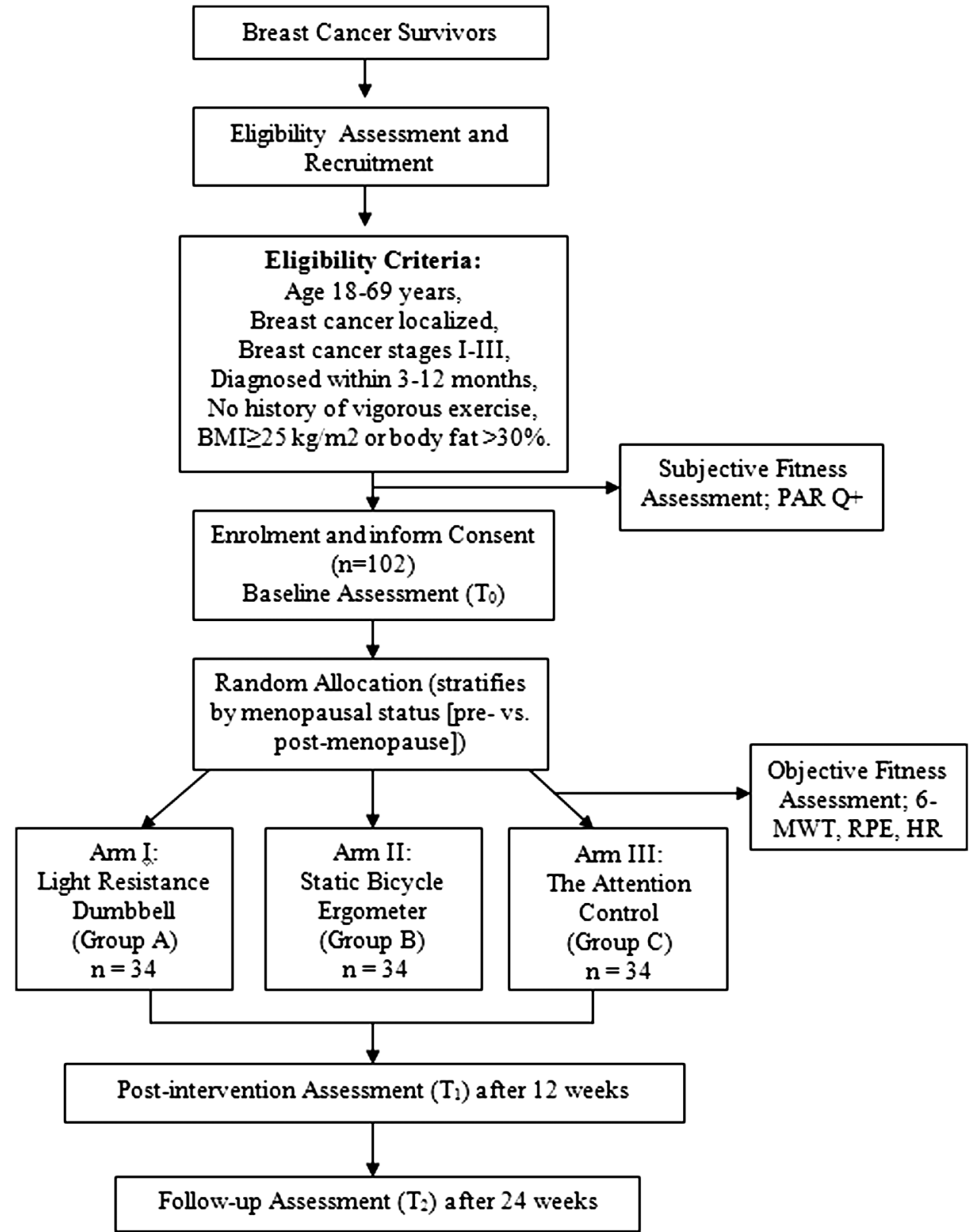

Figure 2 Flow chart of the study recruitment of the participants. 6-MWT, 6-min walk test; BMI, body mass index; HR, heart rate; PAR Q+, Physical Activity Readiness Questionnaire; RPE, ratings of perceived exertion.

The revised Physical Activity Readiness Questionnaire and additional questions (PAR-Q+) that screens for exercise contraindications and precautions will be used for the screening. This questionnaire is designed to screen people with underlying medical conditions where exercise is contraindicated such as musculoskeletal, cardiovascular/respiratory, and other metabolic conditions. ${ }^{42}$ Participants who satisfy the health and fitness screening will then be allocated into the intervention groups.

\section{Randomisation and blinding}

The random allocation of participants into three intervention arms will be based on 1:1:1 ratio. The randomisation will be done by a computer software programme called Research Randomizer (available at https://www.randomizer.org/). Baseline assessments will be done before participants are assigned to the groups. After stratification by menopausal status (premenopause versus post-menopause), participants will be assigned randomly to one of the three groups (light resistance dumbbell, static bicycle ergometer, and control). To enable the stratification, two separate randomisation lists will be made before the random assignment. Blinding the key researcher(s) and the participants will not be possible in our case due to the nature of the study. However, the research assistants who 
Table 1 Criteria for participation in the study

\begin{tabular}{|c|c|}
\hline Inclusion criteria & Exclusion criteria \\
\hline $\begin{array}{l}\text { Female breast cancer } \\
\text { patient aged } 18-69 \text { years }\end{array}$ & $\begin{array}{l}\text { Metastasised and/or stage IV } \\
\text { breast cancer }\end{array}$ \\
\hline $\begin{array}{l}\text { Breast cancer diagnosis } \\
\text { confirmed by histology }\end{array}$ & $\begin{array}{l}\text { Pregnant or intending to } \\
\text { become pregnant during } \\
\text { intervention period }\end{array}$ \\
\hline $\begin{array}{l}\text { Localised breast cancer } \\
\text { treated within past } 3- \\
12 \text { months }\end{array}$ & $\begin{array}{l}\text { Underlying medical conditions } \\
\text { such as musculoskeletal } \\
\text { conditions }\end{array}$ \\
\hline $\begin{array}{l}\text { Patients having good } \\
\text { performance status } \\
(\text { ECOG-1) }\end{array}$ & $\begin{array}{l}\text { Plan or intention to have } \\
\text { reconstructive surgery within } \\
\text { intervention period }\end{array}$ \\
\hline $\begin{array}{l}\text { Absence of any disorder } \\
\text { contraindicating exercise }\end{array}$ & $\begin{array}{l}\text { Previous history of any } \\
\text { physical conditions that make } \\
\text { physical activity impossible }\end{array}$ \\
\hline
\end{tabular}

ECOG, Eastern Cooperative Oncology Group.

will conduct subjective and objective measurements will be blinded.

\section{Fitness assessment}

Prospective participants who pass the health and fitness screening and are assigned into the intervention groups, based on the randomisation, will later be invited to undergo the fitness assessment; each participant will be subjected to a fitness test using the 6-min walk test as follows.

Participants will be instructed to walk along a level surface of a predetermined $6 \mathrm{~m}$ distance as far as they can within the 6-min provided. Before the test, the objective, procedure, and utility of the test findings will be explained to all the participants. ${ }^{43}$ This test has many advantages. It is cost-effective and resembles normal activities of daily living. It is also very easy to use and participants have control over their pace. In addition, it is better tolerated as participants can walk within their endurance limit. ${ }^{44}$ The ratings of perceived exertion (RPE) will be obtained using the Borg RPE 15-grade scale after the 6 -min walk test. ${ }^{45}$ The use of this scale in exercise prescription is recommended to be integrated with heart rate (HR), as strong correlations between them have been reported. ${ }^{46}$ HR of the participants will be taken after $5 \mathrm{~min}$ rest on arrival at the exercise venue. The HR will be taken again after completing the 6-min walk test. The outcomes of RPE and HR will determine the exercise capacity of a participant; hence an exercise regimen will be prescribed according to the fitness level as identified using the RPE and HR readings.

\section{Interventions}

Participants in the exercise therapy groups will engage in the exercise regimen three times weekly for 12 consecutive weeks, spending nearly $60 \mathrm{~min}$ per exercise session. The training session will be done one-on-one as the intervention is primarily tailored towards an individualised exercise programme. An attendance record will be taken for all the participants at the end of each exercise session. Those who are absent in any session will be contacted and their reasons documented. A provision to make up for the lost session will thereafter be provided on the following day or at the end of the intervention period. Each exercise session will comprise a 5-10 min warm up period, 30-45 min exercise training, and 5-10 min cool down exercises. The warm up and cool down exercises will include light aerobic activities and stretching.

Group A (light resistance dumbbell): This group will participate in resistance exercise using a light resistance dumbbell, three times per week. The basic position assumed during the exercise will involve the subject standing straight on a flat surface with both feet at shoulder width distance. The upper body will be bent forward with the back stretched, and the knees also bent to assume and maintain a half crouching position. The programme is made up of 12 exercises as shown in table 2. Details of the exercise programme have been reported elsewhere. ${ }^{38}$ In this position, the dumbbell will be gripped firmly in both hands and the wrists bent into the flexed position. This style will be maintained always while holding the dumbbells since shaking the grip may injure the muscles. All movements must be done slowly and continuously. If participants feel tired they will rest for $30 \mathrm{~s}$ to $1 \mathrm{~min}$, but not more than $2 \mathrm{~min}$. The initial session will be 5-10 repetitions of each exercise depending on individual ability. This will be increased by five repetitions after the third, sixth and ninth weeks to reach a maximum of 25 repetitions towards the end of the protocol. Each session will span between 45-60 min including resting periods between the sessions. The session will begin with warm-up exercises including stretching, followed by the resistance training, and ending with a cool down activity. Participants will move the dumbbells within $2 \mathrm{~s}$ for the eccentric motions and

Table 2 Resistance exercise (dumbbell) programme

\begin{tabular}{ll}
\hline Steps & Description \\
\hline 1 & Push-up and pull-down \\
\hline 2 & Pull-up and push-forward \\
3 & Squat with dumbbell \\
4 & Abdomen twist \\
5 & Chest expand \\
6 & Butterfly \\
7 & Pull up and push down \\
8 & Arm movements \\
99 & Abdomen stretch \\
10 & Arm swing \\
11 & Body swing \\
12 & Shoulder stretch \\
\hline
\end{tabular}


$3 \mathrm{~s}$ for the concentric motions. Participants will receive training beforehand on the correct posture, appropriate movement and speed to carry out the exercise training to ensure they perform the exercise correctly.

Group B (static bicycle ergometer): This group will participate in aerobic exercise training using a static bicycle ergometer, three times per week. The subjects will perform the exercise at sub-maximal intensity that will be determined from the initial fitness assessment. At the beginning, subjects will exercise at 90 watts (W) for $20 \mathrm{~min}$, which will be increased by $30 \mathrm{~W}$ after the third, sixth and ninth weeks to reach a maximum $150 \mathrm{~W}$ towards the end of the protocol. The exercise session will begin with light aerobic warm-up and stretches followed by cycling at 60 revolutions per minute (rpm) and ends with a cool down activity.

Group $C$ (control): This group will be advised to perform their daily activities as usual and continue with the care regimen as advised by their oncologist. Participants in this group will be offered a 1-month supervised exercise at the end of the intervention programme.

\section{Outcomes and assessments}

All participants will be assessed at three different periods: one at baseline, and then at 12 weeks after intervention, and another at 12 weeks follow-up, respectively. The baseline measures will include sociodemographic data, physical activity, quality of life, anthropometric characteristics, and diet history. Post-intervention and during follow-up, all primary (physical activity and quality of life domains) and secondary (BMI, body composition, waist-to-hip ratio, and total caloric intake) outcome variables will be reassessed.

Demographic data: A brief demographic form will be used to obtain the participants' sociodemographic variables such as age, ethnicity, marital status, employment status, educational status, and level of income. Other matters to be assessed include type of cancer, stage at diagnosis, duration after diagnosis, and the types of treatment received.

Physical activity: The physical activity outcomes will be surveyed with the International Physical Activity Questionnaire-short-form (IPAQ-SF) version. The IPAQ-SF was developed with the purpose of providing an instrument for physical activity estimates that can be comparable across the nations. The tool may either be self- or interviewer-administered and has been tested for validity and reliability across 12 countries. ${ }^{47}$ The Malay version of the tool used in this study was adopted and translated from English, and has been validated according to international practice. ${ }^{48}$ IPAQ-SF is made up of seven items that elicits the duration and frequency of vigorous- and moderate-intensity activities and walking that took at least $10 \mathrm{~min}$ in a bout during the previous week. This involves all the daily life activities such as household chores, occupational and leisure time activities, as well as transportation. The time spent in sitting down is also assessed in order to determine the extent of the sedentary lifestyle of respondents.

The data obtained will be scored according to the 'Guidelines for data processing and analysis of the International Physical Activity Questionnaire (IPAQ)Short and Long Forms'. Briefly, based on the intensities, the physical activity measured will be converted to metabolic equivalent (MET) score by multiplying the number of minutes and days of a given activity by its corresponding MET value. The MET values for vigorous intensity, moderate intensity, and walking are 8.0, 4.0, and 3.3 respectively. Therefore, MET-min/week for each of the physical activities are computed as follows:

1. Vigorous intensity (MET-min/week)=vigorous intensity minutes $\times 8.0 \times$ vigorous intensity days

2. Moderate intensity $\quad($ MET-min $/$ week $)=$ moderate intensity minutes $\times 4.0 \times$ moderate intensity days

3. Walking $($ MET-min/week $)=$ walking minutes $\times 3.3 \times$ walking days

4. Total physical activity (MET-min/week)=vigorous intensity (MET-min/week)+moderate intensity (MET-min/week)+walking (MET-min/week).

The scores obtained will be categorised into low, moderate, and high levels of physical activity according to the IPAQ instruction for physical activity classification. Further information about the IPAQ-SF can be accessed at http://www.ipaq.ki.se/scoring.pdf.

Quality of life: The health-related quality of life outcomes will be assessed using the Functional Assessment for Cancer Therapy-Breast (FACT-B) V.4. This version was specifically developed to assess health-related quality of life in breast cancer patients. The tool is made up of 44 items on a 5-point Likert scale. The response options range from 'Not at all' to 'Very much' and respondents are encouraged to answer all items by choosing a response that is most applicable to them. The 44 items are divided into five scales that comprise physical wellbeing, social/family well-being, emotional well-being, functional well-being, and breast cancer specific concerns. The raw scores will be computed into the above scales according to the recommendations for using FACIT (Functional Assessment of Chronic Illness Therapy) tools. ${ }^{49}$ The original English version has been translated into Malay language in line with the best practices for cross-cultural adaptation and translation and the psychometric properties tested. ${ }^{50}$

\section{Anthropometry}

The anthropometric outcomes will be assessed according to standard guidelines for anthropometric assessment. $^{51}$ The measurements will be taken at least two times on every site, and a third measurement will be taken in cases where a difference of $>0.5 \mathrm{~cm}$ is observed between the first and second readings for the height, waist circumference, and hip circumference.

Weight, height, and BMI: The body weight will be measured using a digital weight and height scale (SECA TZ-160, Hamburg, Germany). The values obtained will 
be approximated to the nearest $0.1 \mathrm{~kg}$. The height of participants will be measured (to the nearest $0.1 \mathrm{~m}$ ) using the same weight and height scale (SECA TZ-160, Hamburg, Germany). The BMI value will then be estimated from the standard calculation of weight divided by height ${ }^{2}$ (ie, $\mathrm{kg} / \mathrm{m}^{2}$ ).

Waist circumference, hip circumference and WHR: Waist and hip circumferences will be measured (to the nearest $0.1 \mathrm{~cm}$ ) using an inelastic tape rule (Butterfly, Shanghai, China). The midpoint between the iliac crest and the lowest rib will be considered as the reference point for measuring the waist circumference. The area with maximum protrusion of buttocks on standing with the feet close together will be considered as the reference point for the hip circumference measurement. The values recorded for waist and hip circumferences will be used to determine the waist-to-hip ratio.

\section{Body composition}

Each participant's body composition, including body fat percentage and fat free mass, will be assessed using the body composition analyser (Tanita SC-330, Tokyo, Japan) which uses bioelectrical impedence analysis. This protocol uses electrical impedence to compute an estimate of the total body water, fat-free mass, and body fat. The body composition analyser, Tanita SC-330, provides estimated values similar to the values obtained by dual-energy X-ray absorptiometry (DXA) and MRI methods. ${ }^{52}$ The equipment has wide applicability including assessing the effectiveness of individual nutrition and exercise programmes, both for health and physical fitness. The equipment is simple to use and measurements can be easily and quickly taken, hence reducing patient inconvenience during the measuring process.

\section{Diet history}

The diet history will be assessed using a 3-day food diary. Participants will be required to record their food (including snacks) and beverage consumption in a typical day. The record will cover one weekend and two weekdays. The values obtained will be analysed to determine the total caloric intake of the participants. The outcomes to be assessed and the tools to be used for the assessment are presented in table 3 .

\section{Sample size}

The principal aims of this intervention are to compare changes obtained in physical activity estimates and overall quality of life from baseline to week 24, between the exercise groups (A and B) and control group (C). The literature on physical activity intervention programmes among breast cancer survivors has shown small to large effects on physical activity level and overall quality of life. Based on the effect size, our choice for calculating sample size was 'moderate' due to the preliminary nature of this study. Besides, to the best of our knowledge to date, there has been no study in Malaysia that assesses the feasibility of carrying out an
Table 3 Measurement tools and assessment outcomes

\begin{tabular}{|c|c|}
\hline Measurement tool & Assessment outcome \\
\hline \multicolumn{2}{|l|}{ Primary end point } \\
\hline IPAQ-SF & Physical activity estimate \\
\hline FACT-B & Components of quality of life \\
\hline \multicolumn{2}{|l|}{ Secondary end point } \\
\hline $\begin{array}{l}\text { Body composition } \\
\text { analyser Tanita SC-330 }\end{array}$ & Body composition and BMI \\
\hline Measuring tape & Waist and hip circumference \\
\hline 3-day food diary & Total caloric intake \\
\hline \multicolumn{2}{|l|}{ Other assessments } \\
\hline Demographic data form & $\begin{array}{l}\text { Sociodemographic } \\
\text { characteristics }\end{array}$ \\
\hline $\begin{array}{l}\text { Breast cancer } \\
\text { characteristics }\end{array}$ & $\begin{array}{l}\text { Family history, type of breast } \\
\text { cancer, stage at diagnosis of } \\
\text { the disease, and duration } \\
\text { after diagnosis }\end{array}$ \\
\hline Treatment record & $\begin{array}{l}\text { Type of surgery, } \\
\text { chemotherapy, radiotherapy, } \\
\text { and number of cycles }\end{array}$ \\
\hline
\end{tabular}

BMI, body mass index; FACT-B, Functional Assessment for Cancer Therapy-Breast; IPAQ-SF, International Physical Activity Questionnaire-short-form.

individualised exercise programme such as the use of light resistance dumbbells among breast cancer survivors. In addition, according to our proposed statistical analyses, we need 28 participants per group which gives a sample size of 84 participants. However, to cater for dropout, a $20 \%$ withdrawal rate was considered giving a total of 102 participants. Furthermore, a systematic review and meta-analysis data have reported a range of sample sizes used in physical activity trials, ranging from 6-319 participants per intervention group and 4-322 participants per control group. ${ }^{53}$

\section{Statistical analysis}

Statistical Package for Social Sciences V.22.0 (SPSS Chicago, USA) will be employed to analyse the obtained data using per protocol analysis as follows. Two-way analysis of variance (ANOVA) will be used for baseline comparisons of demographic variables. For comparison of change scores in randomisation groups, the primary outcomes (physical activity estimate and quality of life domains) will be reported as independent samples. Depending on the normality of data distribution, change scores for secondary outcomes (body composition, BMI, total caloric intake, waist-to-hip ratio) for individual variables will be determined and compared between intervention groups by employing appropriate procedures for parametric or non-parametric analysis. To test the aim of the study, a time by group interaction term will be employed. Also for time $\left(T_{0}, T_{1}\right.$ and $\left.T_{2}\right)$ and group (A, B and $\mathrm{C}$ ) allocation being fixed factors, random effect mixed modelling will be used. Overall, to control for sociodemographic variables, repeated 
measure ANOVA will be used to determine differences in both primary and secondary outcomes between groups over time. Analysis of covariance (ANCOVA) will be used to determine the differences in postrandomisation outcomes among study groups (adjusting for pre-randomisation values and other factors). $\alpha$ Level of significance will be set at a probability value of $\mathrm{p} \leq 0.05$.

\section{Ethical approval}

The approval of this study has been granted by the Human Research Ethics Committee of Universiti Sains Malaysia.

\section{DISCUSSION}

The number of breast cancer survivors will continue to increase as a result of improvements in detection and treatment modalities. Unfortunately the cancer itself and its associated side effects will continue to disturb breast cancer survivors in the long term. ${ }^{54}$ Exercise has been shown to be beneficial in relieving some of the adverse effects of cancer and its treatment. ${ }^{55}$ However, whether exercise participation can be encouraged among breast cancer survivors by using less sophisticated gadgets such as the light resistance dumbbell has not been studied.

This study will add to the existing pool of information in the emerging area of exercise therapy among breast cancer survivors in several ways: first, through evaluation of physical activity levels and quality of life components following light resistance dumbbell exercise; second, the use of light resistance dumbbells in exercise therapy training and the addition of premenopausal women will contribute to this expanding field; third, inclusion of 'early' breast cancer survivors, and the 12-week follow-up period after intervention to test the sustainability effects of the exercise, will also add to the available literature.

Evidence continues to emerge on the potential benefits of physical activity intervention programmes on the levels of physical activity and quality of life outcomes in breast cancer survivors. This is observed from the increasing number of exercise therapy trials being recorded in clinicaltrials.gov. ${ }^{56}$ According to the current literature, physical activity intervention programmes after breast cancer treatment have small to large effects on breast cancer-specific concerns, symptoms and side effects, fatigue, aerobic fitness, physical activity level, and overall quality of life. Similarly, during the course of treatment these effects are reported on bodyweight, aerobic fitness, functional quality of life, anxiety, and self-esteem. ${ }^{53}$ Data reported elsewhere may not necessarily be applicable to Malaysian breast cancer survivors. Currently, however, there is a dearth of information on exercise intervention in Malaysia and no physical activity data are available among breast cancer survivors. Moreover, to the best of our knowledge, no study has been conducted using light resistance dumbbell exercise among breast cancer survivors.

Studies that deal with topical issues on the specific exercise demands of breast cancer survivors are lacking. Recently, however, a study conducted among rural breast cancer survivors on exercise preference reported that the participants preferred face-to-face exercise counselling both pre- and post-treatment. ${ }^{57}$ The present study addresses this need, offering face-to-face exercise instruction with the aim of achieving sustainability of the programme. In the process, it will be explained that participation in exercise does not mean going to the gymnasium and/or using sophisticated and expensive gadgets that involves extra costs. Most of the early studies have used sophisticated gadgets for resistance exercises. In contrast, this study involves the use of light resistance dumbbells which are very comfortable to grasp and hold while working the required muscle group(s). According to Milne et $a l^{58}$ breast cancer survivors attached importance to exercise as a vehicle for health promotion irrespective of whether they participate or not; this further supports the need to encourage exercise participation through an exercise programme that is feasible and affordable. Furthermore, an intervention that is tailored towards an individual's current physical activity ability may be more widely accepted. This will enhance participation in physical activity among breast cancer survivors, particularly those lacking access to modern sophisticated facilities.

Determining the right time for intervention is as important as the intervention itself. An earlier study ${ }^{59}$ defined the 'teachable moment' as a critical moment when lifestyle behaviours of survivors change during the course of treatment. It is presently not clear when is the best time for breast cancer survivors to engage in physical activity. Holding on to the 'teachable moment', change in the behaviour of breast cancer survivors will be promoted by participation in supervised exercise training. In addition, since the exercise programme creates a calming and assuring condition, the change will last for a longer duration of time. Therefore, early intervention is useful because breast cancer survivors may have higher chances of accommodating psychological problems associated with their diagnosis for a longer duration compared to other cancer survivors. They may also be more interested in participating in the intervention programme. ${ }^{60}$ All of these factors support the need for early intervention. This is in agreement with our inclusion phrase of 'early breast cancer survivors'. The 'early breast cancer survivors' involve women diagnosed with breast cancer and receiving cancerrelated treatments within the first 12 months after diagnosis. This is particularly true as most of the exercise trials for breast cancer survivors included participants from 6 months to 10 years and above after the treatment. ${ }^{61}$ We therefore strongly believe that to ride on the crest of the teachable moment, early breast cancer survivors need to be included. 
One unique element of this study is the inclusion of a 12-week post-intervention follow-up period. The follow-up after completion of the intervention will enable evaluation of long-term adherence to the exercise. Long-term adherence will provide the sustainability of exercise participation even after the exercise intervention programme is completed. Studies on exercise interventions among cancer cases have reported $>80 \%$ adherence rates, but these studies did not explore whether the advantages of the exercise intervention lasted in the long term after termination of the study. ${ }^{62} 63$ The gains from exercise mostly fade away because participants fail to continue the exercise regularly after termination of the study. Therefore, there is a need to re-examine long-term exercise adherence following termination of the study. Numerous exercise interventions among breast cancer survivors seek to bring about change in lifestyle behaviour from a sedentary to a physically active one, but there is no sufficient evidence to demonstrate exercise adherence following an exercise intervention.

Thus, the present study will find out if the precise gains of exercise succeeding a 12-week follow-up can perpetuate in the absence of active enrolment and supervision of exercise intervention by investigators. Data on the diet and physical activity patterns and effects, which will be obtained at the time of this study, may supply useful knowledge that may be applied in the planning of future studies. This will contribute immensely in achieving the long-term goal of adherence to exercise among the growing population of breast cancer survivors.

\section{Handling editor Seye Abimbola}

Contributors AAR, BVKM and WAMWM planned and coordinated the study. AAR and BVKM conducted the study. AAR drafted the manuscript. All authors contributed to the protocol design and edited the manuscript for intellectual content. All authors read and approved the final version of the manuscript.

Funding This study is supported by the Universiti Sains Malaysia RUT Grant initiative, and AAR is supported by the Malaysian International Scholarship Award.

\section{Competing interests None declared.}

Ethics approval Human Research Ethics Committee of Universiti Sains Malaysia.

Provenance and peer review Not commissioned; externally peer reviewed.

Data sharing statement No additional data are available.

Open Access This is an Open Access article distributed in accordance with the Creative Commons Attribution Non Commercial (CC BY-NC 4.0) license, which permits others to distribute, remix, adapt, build upon this work noncommercially, and license their derivative works on different terms, provided the original work is properly cited and the use is non-commercial. See: http:// creativecommons.org/licenses/by-nc/4.0/

\section{REFERENCES}

1. Howlader N, Noone AM, Krapcho M, et al., eds. SEER Cancer Statistics Review, 1975-2009 (Vintage 2009 Populations). Bethesda, MD: National Cancer Institute. http://seer.cancer.gov/csr/1975 2009_pops09 (accessed 21 Aug 2012).
2. Bhoo-Pathy $\mathrm{N}$, Yip $\mathrm{CH}$, Hartman $\mathrm{M}$, et al. Breast cancer research in Asia: adopt or adapt Western knowledge? Eur J Cancer 2013;49:703-9.

3. Youlden DR, Cramb SM, Yip CH, et al. Incidence and mortality of female breast cancer in the Asia-Pacific region. Cancer Biol Med 2014;11:101-15.

4. Makari-Judson $\mathrm{G}$, Judson $\mathrm{CH}$, Mertens WC. Longitudinal patterns of weight gain after breast cancer diagnosis: observations beyond the first year. Breast $J$ 2007;13:258-65.

5. Morrow GR, Shelke AR, Roscoe JA, et al. Management of cancer-related fatigue. Ca Investigation 2005;23:229-39.

6. Schmitz KH, Courneya KS, Matthews C, et al. American College of Sports Medicine roundtable on exercise guidelines for cancer survivors. Med Sci Sports Exerc 2010;39:1409-26.

7. Harrington S, Padua D, Battaglini C, et al. Upper extremity strength and range of motion and their relationship to function in breast cancer survivors. Physiother Theory Pract 2013;29:513-20.

8. Dominick SA, Madlensky L, Natarajan L, et al. Risk factors associated with breast cancer-related lymphedema in the WHEL Study. J Cancer Surv 2013;7:115-23.

9. Galiano-Castillo N, Ariza-Garcia A, Cantarero-Villanueva I, et al. Depressed mood in breast cancer survivors: associations with physical activity, cancer-related fatigue, quality of life, and fitness level. Eur J Oncol Nurs 2013;18:206-10.

10. Chen Z, Maricic M, Bassford TL, et al. Fracture risk among breast cancer survivors: results from the women's health initiative observational study. Arch Intern Med 2005;165:552-8.

11. Irwin ML, McTiernan A, Baumgartner RN, et al. Changes in body fat and weight after a breast cancer diagnosis: influence of demographic, prognostic, and lifestyle factors. J Clin Oncol 2005;23:774-82.

12. Thomson CA, Thompson PA, Wright-Bea J, et al. Metabolic syndrome and elevated C-reactive protein in breast cancer survivors on adjuvant hormone therapy. J Womens Health (Larchmt) 2009;18:2041-7.

13. Demark-Wahnefried W, Peterson BL, Winer EP, et al. Changes in weight, body composition, and factors influencing energy balance among premenopausal breast cancer patients receiving adjuvant chemotherapy. J Clin Oncol 2001;19:2381-9.

14. Battaglini CL, Mihalik JP, Bottaro M, et al. Effect of exercise on the caloric intake of breast cancer patients undergoing treatment. Brazilian J Med Biol Res 2008;41:709-15.

15. Whiteman MK, Hillis SD, Curtis KM, et al. Body mass and mortality after breast cancer diagnosis. Cancer Epidemiol Biomarkers Prev 2005;14:2009-14.

16. Dimeo FC. Effects of exercise on cancer-related fatigue. Cancer 2001;92(6 Suppl):1689-93.

17. Courneya KS, Friedenreich CM. Physical exercise and quality of life following cancer diagnosis: a literature review. Ann Behav Med 1999;21:171-9.

18. Freedman RJ, Aziz N, Albanes D, et al. Weight and body composition changes during and after adjuvant chemotherapy in women with breast cancer. J Clin Endocrinol Metab 2004;89:2248-53.

19. Battaglini C, Bottaro M, Dennehy C, et al. The effects of an individualized exercise intervention on body composition in breast cancer patients undergoing treatment. Sao Paulo Med J 2007;125:22-8.

20. Fong DYT, Ho JWC, Hui BPH, et al. Physical activity for cancer survivors: meta-analysis of randomised controlled trials. BMJ 2012;344:1-14.

21. Ferrer R, Huedo-Medina TB, Johnson BT, et al. Exercise interventions for cancer survivors: a meta-analysis of quality of life outcomes. Ca Epidemiol Biomarkers Prev 2011;41:32-47.

22. Sabiston $\mathrm{CM}$, Brunet $\mathrm{J}$. Reviewing the benefits of physical activity during cancer survivorship. Am J Lifestyle Med 2012;6:167-77.

23. Dimeo F, Rumberger BG, Keul J. Aerobic exercise as therapy for cancer fatigue. Med Sci Sports Exerc 1998;30:475-8.

24. Midtgaard J, Rorth M, Stelter R, et al. The group matters: an explorative study of group cohesion and quality of life in cancer patients participating in physical exercise intervention during treatment. Eur J Ca Care 2006;15:25-33.

25. Gjerset GM, Fossa SD, Courneya KS, et al. Exercise behavior in cancer survivors and associated factors. J Ca Survivorship 2011;5:35-43.

26. Irwin ML, Varma K, Alvarez-Reeves M, et al. Randomized controlled trial of aerobic exercise on insulin and insulin-like growth factors in breast cancer survivors: the Yale Exercise and Survivorship Study. Cancer Epidemiol Biomarkers Prev 2009;18:306-13.

27. Knobf MT, Insogna K, DiPietro L, et al. An aerobic weight-loaded pilot exercise intervention for breast cancer survivors: bone 
remodeling and body composition outcomes. Biol Res Nurs 2008;10:34-43.

28. Twiss JJ, Waltman NL, Berg K, et al. An exercise intervention for breast cancer survivors with bone loss. J Nurs Scholarsh 2009;41:20-7.

29. De Backer IC, Van Breda E, Vreugdenhil A, et al. High-intensity strength training improves quality of life in cancer survivors. Acta Oncol 2007;46:1143-51.

30. Ahmed RL, Thomas W, Yee D, et al. Randomized controlled trial of weight training and lymphedema in breast cancer survivors. J Clin Oncol 2006;24:2765-72.

31. Schmitz $\mathrm{KH}$, Ahmed RL, Troxel A, et al. Weight lifting in women with breast-cancer-related lymphedema. N Engl J Med 2009;361:664-73.

32. Cormie P, Pumpa K, Galvao DA, et al. Is it safe and efficacious for women with lymphedema secondary to breast cancer to lift heavy weights during exercise: a randomised controlled trial. J Cancer Surv 2013;7:413-24.

33. Cheema BS, Kilbreath SL, Fahey PP, et al. Safety and efficacy of progressive resistance training in breast cancer: a systematic review and meta-analysis. Breast Cancer Res Treat 2014;148:249-68.

34. Suzuki M. Health promotion by dumbbell exercise. Bull Int Health Sci Univ Tsukuba 2003;26:1-6 (in Japanese).

35. Matsuo T, Suzuki M. Effect of dumbbell exercise with and without energy restriction on resting metabolic rate, diet-induced thermogenesis and body composition in mildly obese women. Asia Pac J Clin Nutr 1999:8:136-41.

36. Matsuo T, Suzuki M. Effects of low-calorie diet therapy with and without dumbbell exercise on body fat reduction in obese young women. J Clin Biochem Nutr 1998;25:49-62.

37. Kang $\mathrm{H}$, Matsuo $\mathrm{T}$, Suzuki M. Effects of light resistance exercise using dumbbells and rubber band with mild energy restriction on body composition and physical fitness in obese Korean women. Asia Pac J Clin Nutr 2004;13:242-7.

38. Kato $\mathrm{Y}$, Sawada A, Numao S, et al. Effect of light resistance exercise after ingestion of a high-protein snack on plasma branched-amino acid concentrations in young adult females. $J$ Nutr Sci Vitaminol 2009;55:106-11.

39. Kato Y, Numao S, Miyauchi R, et al. Effect of intermittent blood volume fluctuation of light resistance exercise after ingestion of the high-protein snacks on plasma branched-amino acid concentrations in young adult females. J Nutr Sci Vitaminol 2010;56:255-9.

40. Doi T, Matsuo T, Sugawara M, et al. New approach for weight reduction by a combination of diet, light resistance exercise and the timing of ingesting a protein supplement. Asia Pacific J Clin Nutr 2001;10:226-32.

41. National Coalition for Cancer Survivorship (NCCS, 2004). Glossary c. 1994-2004. http://canceradvocacy.org/resources/glossary.aspxtop (accessed 7 Mar 2015).

42. Warburton DER, Jamnik VK, Bredin SSD, et al., on behalf of the PAR-Q+ Collaboration. The physical activity readiness questionnaire (PAR-Q+). Health Fitness J Can 2011;4:3-23.

43. Guyatt GH, Sullivan MJ, Thompson PJ, et al. The 6-minute walk test: a new measure of exercise capacity in patients with chronic heart failure. Can Med Assoc J 1985;132:919-23.

44. Enright PL. The six-minute walk test. Respiratory Care 2003;48:783-5.

45. Borg GA. Psychophysical bases of perceived exertion. Med Sci Sport Exer 1982;14:377-81.
46. Mihevic PM. Sensory cues for perceived exertion: a review. Med Sci Sport Exer 1981;13:150-63.

47. Craig CL, Marshall AL, Sjöström M, et al. International physical activity questionnaire: 12 -country reliability and validity. $\mathrm{Med} \mathrm{Sci}$ Sport Exer 2003;35:1381-95.

48. Teh $\mathrm{CH}$, Lim KK, Chan YY, et al. The prevalence of physical activity and its associated factors among Malaysian adults: findings from the National Health and Morbidity Survey 2011. Public Health 2014;128:416-23.

49. Brady MJ, Cella DF, Mo F, et al. Reliability and validity of the functional assessment of cancer therapy-breast quality of life instrument. J Clin Oncol 1997;15:974-86.

50. Redhwan AA, Md Idris MN, Zaleha MI, et al. Quality of life among women with breast cancer from Universiti Kebangsaan Malaysia medical centre, Malaysia. J Comm Health 2008;14:46-55.

51. World Health Organization. Obesity: preventing and managing the global epidemic of obesity. Report of WHO consultation on obesity; WHO technical report series. Geneva, 2011:894.

52. Tanita Manual. Body composition analyzer SC-330: Instruction manual. Tokyo, Japan: 2004, Tanita Corporation. http://www.tanita. co.jp

53. Speck RM, Courneya KS, Masse LC, et al. An update of controlled physical activity trials in cancer survivors: a systematic review and meta-analysis. J Ca Survivorship 2010;4:87-100.

54. Wong P, Muanza T, Hijal T, et al. Effect of exercise in reducing breast and chest-wall pain in patients with breast cancer: a pilot study. Current Oncol 2012;19:e129-135.

55. Friedenreich CM. Physical activity and cancer prevention: from observational to intervention research. Cancer Epidemiol Biomarkers Prev 2001;10:287-301.

56. Dieli-Conwright CM, Mortimer JE, Schoeder ET, et al. Randomized controlled trial to evaluate the effects of combined progressive exercise on metabolic syndrome in breast cancer survivors: rationale, design, and methods. BMC Cancer 2014;14:238.

57. Rogers LQ, Markwell SJ, Verhulst S, et al. Rural breast cancer survivors: exercise preferences and their determinants. Psycho-Oncol 2009;18:412-21.

58. Milne HM, Guilfoyle A, Gordon S, et al. Personal accounts of exercise and quality of life from the perspective of breast cancer survivors. Qual Life Res 2007;16:1473-81.

59. Demark-Wahnefried W, Aziz NM, Rowland JH, et al. Riding the crest of the teachable moment: promoting long-term health after the diagnosis of cancer. J Clin Oncol 2005;23:5814-30.

60. McBride CM, Clipp E, Peterson BL, et al. Psychological impact of diagnosis and risk reduction among cancer survivors. Psycho-Oncol 2000;9:418-27.

61. Spark LC, Reeves MM, Fjeldsoe BS, et al. Physical activity and/or dietary interventions in breast cancer survivors: a systematic review of the maintenance of outcomes. J Cancer Surviv 2013;7:74-82.

62. Latka RN, Alvarez-Reeves M, Cadmus L, et al. Adherence to a randomized controlled trial of aerobic exercise in breast cancer survivors: the Yale Exercise and Survivorship Study. J Cancer Surviv 2009;3:148-57.

63. Irwin ML, Cadmus L, Alvarez-Reeves M, et al. Recruiting and retaining breast cancer survivors into a randomized controlled exercise trial: the Yale Exercise and Survivorship Study. Cancer 2008;112(11 Suppl):2593-606. 